Pensamiento Crítico N. ${ }^{\circ}$ 9, pp. 151-170

\title{
Marco para el análisis de la seguridad social en el Perú
}

\author{
Mg. César A. Sanabria Montañez
}

\section{RESUMEN}

El presente artículo es un avance del estudio que se desarrolla en el Instituto de Investigaciones Económicas de la Universidad Nacional Mayor de San Marcos, pretende ser un marco de referencia del estudio sobre seguridad social, por ello se explora el contexto económico social peruano, a la luz de la contradicción existente entre crecimiento económico y pobreza; asimismo, se examina las principales características del empleo, el crecimiento demográfico y los retos futuros para la sociedad en términos de inversión en las personas a través de la seguridad social; se revisa también el estado del arte de la seguridad social en salud y de pensiones en el Perú. Finalmente, se presenta el marco conceptual de lo que se entiende por seguridad social desde el enfoque económico.

Palabras clave: Seguridad social, salud, protección social, riesgo social, política social.

\section{ABSTRACT}

The present article is an advance of the study that develop in the Institute of Economic Investigations of the National University Main of Saint Marcos, pretends to be a frame of reference of the study on social security, thus it explores the economic 


\section{César A. Sanabria Montañez}

context social Peruvian, to the light of the existent contradiction between economic growth and poverty; likewise examines the main characteristics of the employment, the demographical growth and the future the future changes for the society in terms of investment in the people through the social security; review also the state of the art of the social security in health and of pensions in the Peru. Finally presents the conceptual frame of what understand by social security from the economic approach.

Keywords: Social security, health, social protection, social risk, social politics

\section{EL CONTEXTO ECONÓMICO SOCIAL EN PERÚ}

La economía peruana actualmente se encuentra en un ciclo expansivo, en los últimos años ha tenido una actividad económica con tasas altas de crecimiento. "En 2007, el Producto Bruto Interno se expandió en 9,0\%, la tasa de crecimiento más alta desde 1994. Con este resultado, a diciembre de 2007, se ha experimentado un crecimiento continuo de 78 meses. Al igual que en 2006, esta expansión se ha explicado, fundamentalmente, por el dinamismo del consumo e inversión privados, los que impulsaron un incremento de la demanda interna de $11,7 \%$ (1,7 puntos porcentuales por encima de lo registrado en 2006)" 1 . Ello debido al favorable clima económico internacional (con altas tasas de crecimiento y precios altos de nuestros principales productos de exportación), y en el orden interno dentro de un clima de confianza de los agentes, un entorno macroeconómico de estabilidad de precios, aumento del crédito y manejo del gasto público.

Por una parte, el dinamismo del consumo privado en 2007, se explica por el mayor poder adquisitivo de la población debido al incremento del empleo formal de forma descentralizada (8,3\% en empresas de 10 a más trabajadores, a nivel nacional), a la mayor oferta crediticia (créditos de consumo se incrementaron en $37 \%$ ) y a las expectativas optimistas de los consumidores respecto al desempeño de la economía 2 .

En el año 2007, la proporción de personas que participan activamente en el mercado laboral (tasa de actividad) se incrementó a 70,0\% y la tasa desempleo ha tenido un ligero estancamiento en el nivel de 4,5\%. Sin embargo, una mirada detallada a las cifras del empleo, permite llamar la atención en torno a la calidad del empleo generado;

1 Marco Macroeconómico Multianual 2009 - 2011. MEF. Mayo de 2008. Ver también la misma explicación del crecimiento en estos últimos años en la Memoria Anual 2006 del BCRP. p. 15.

2 Ibídem. p. 38. 


\section{Marco para el análisis de la seguridad social en el Perú}

pues, en el 2006, la tasa de subempleo representó el 51,4\% de la PEA y la población adecuadamente empleada el $44 \%$.

Ante el crecimiento de la economía, el mercado laboral peruano ha generado empleo predominantemente precario en su naturaleza, derivado de las persistentes diferencias en la estructura laboral. Las áreas rurales tienen un bajo nivel de asalariamiento $(20 \%)$ y una presencia importante de trabajo familiar no remunerado, a diferencia del área urbana, donde el trabajo asalariado representa la mitad del empleo, y casi no hay trabajo no remunerado. El trabajo independiente o autoempleo existe en ambas zonas, urbana $(40 \%)$ y rural $(52 \%)^{3}$.

Todo ello evidencia que la traslación del crecimiento logrado en la economía hacia los sectores sociales es lento, debido a las causas profundas de la pobreza en el Perú; así se espera que estos indicadores bajen, y es una meta que se ha propuesto el actual Gobierno del Perú para el 2011, así lo señala el discurso presidencial del 28 de julio de 2007, ante el Congreso de la República: "Desde el 2006 al 2011, la inversión privada grande y pequeña, habrá totalizado más de 100 mil millones de dólares de inversión interna. Este año, ya se está invirtiendo más de 20 mil millones privados y, además, la inversión pública que en este 2007, es de 5 mil millones de dólares en agua, electricidad, caminos y apoyo social, llegara a ser un total de 30 mil millones en los 5 años para transformar el rostro humano del Perú. Esto nos llevará a reducir la pobreza desde el $50 \%$ del 2005 al 30\% en el 2011. Las ciudades tendrán menos de $20 \%$ de pobreza y en el campo, donde ahora es casi de 70 la pobreza, bajará a $45 \%$ " 4 . Este propósito de reducción de la pobreza fue reiterado en el discurso presidencial del 28 de julio de 2008: "Reitero mi compromiso, reduciremos la pobreza al $30 \%$ al final de mi mandato" 5 .

Por otra parte, respecto del año 2005, en el año 2006, la demanda interna creció $10,6 \%$, el consumo privado creció $6,5 \%$ (el más alto en los últimos años, y se sustentó en el aumento del ingreso nacional disponible en $11,9 \%$; la expansión del empleo, $7,3 \%$ en las empresas con más de 10 trabajadores, la confianza de los consumidores en la economía nacional y en el mayor acceso al crédito) ${ }^{6}$.

3 MEF: MMM 2007 - 2009.

4 Diario Oficial El Peruano, de fecha 30 de julio de 2007.

5 Diario El Comercio, de fecha 29 de julio de 2008.

6 Ministerio de Economía y Finanzas. MMM. 2007 - 2009. 


\section{César A. Sanabria Montañez}

La población económicamente activa (PEA) ocupada mayor de 14 años es de 11 millones de personas; el $27 \%$ se ubica en Lima Metropolitana; $38 \%$, en el resto urbano del país; y 35\%, en el área rural. La relación entre crecimiento económico y empleo es positiva, pero no proporcional; el PBI creció en $22 \%$ entre el año 2001 y el 2005, el empleo solo creció en $9 \%$.

Ante el crecimiento de la economía, el mercado laboral peruano ha generado empleo predominantemente precario en su naturaleza, derivado de las persistentes diferencias en la estructura laboral. Las áreas rurales tienen un bajo nivel de asalariamiento $(20 \%)$ y una presencia importante de trabajo familiar no remunerado; a diferencia del área urbana, donde el trabajo asalariado representa la mitad del empleo y casi no hay trabajo no remunerado. El trabajo independiente o autoempleo existe en ambas zonas, urbana $(40 \%)$ y rural $(52 \%)^{8}$.

“...En el año 2006, la población económicamente activa (PEA) de Lima Metropolitana, estimada mediante la Encuesta Permanente de Empleo fue de 4,2 millones, mayor en 2,6 por ciento con relación al año 2005. Se registró un incremento en la población ocupada (4,2 por ciento), alcanzando un nivel de 3,9 millones de personas, mientras que la PEA desempleada se redujo en 12,4 por ciento"9.

Asimismo, debemos anotar que el subempleo (por visible y por ingresos) en Lima Metropolitana, alcanzó el año 2006, a 41,2\% de la PEA, mientras que el 50\% está adecuadamente empleado y el $8.8 \%$ se encontraba desempleado ${ }^{10}$.

La PEA ocupada, según estructura de mercado en Lima Metropolitana, nos indica que el $8.1 \%$ de la PEA ocupada trabaja en el sector público; el 51\%, en el sector privado;

el $31.4 \%$ son independientes; $4,0 \%$ son trabajadores familiares no remunerados; y el 5,6\% son trabajadores del hogar, practicantes y otros tipos de empleo (ver Cuadro N. ${ }^{\circ} 1$ ).

7 Ministerio de Economía y Finanzas: Marco Macroeconómico Multianual 2007 - 2009.

8 MEF: MMM 2007 - 2009.

9 Memoria Anual 2006 BCRP. p. 33.

10 MINISTERIO DE TRABAJO Y PROMOCIÓN DEL EMPLEO. Indicadores Laborales. Febrero 2007. Cifras preliminares a octubre de 2006 de la Encuesta de Hogares Especializados en Niveles de Empleo. 


\section{Marco para el análisis de la seguridad social en el Perú}

En ese mismo cuadro, podemos observar que es la microempresa la que capta más empleo, 20,7 por ciento del total de PEA ocupada en Lima Metropolitana, seguida de la Mediana y grande empresa que capta al 17,3\%, y la Pequeña empresa con el 13,2\%.

Cuadro N. ${ }^{\circ}$ 1. Lima Metropolitana: Distribución de la PEA ocupada según estructura de mercado $(\%) .{ }^{11}$

\begin{tabular}{|l|r|r|r|}
\hline \multirow{2}{*}{ ESTRUCTURA DE MERCADO } & \multicolumn{3}{c|}{$\mathbf{2 0 0 6}$} \\
\cline { 2 - 4 } & Hombre & Mujer & Total \\
\hline SECTOR PÚBLICO & 7.9 & 8.3 & 8.1 \\
\hline SECTOR PRIVADO 1/ & $\mathbf{5 8 . 1}$ & $\mathbf{4 1 . 5}$ & $\mathbf{5 1 . 1}$ \\
\hline Microempresa & 23.9 & 16.1 & 20.7 \\
\hline Pequeña Empresa & 14.1 & 11.9 & 13.2 \\
\hline Mediana y grande empresa & 20.1 & 13.4 & 17.3 \\
\hline INDEPENDIENTE & $\mathbf{3 0 . 7}$ & $\mathbf{3 2 . 3}$ & $\mathbf{3 1 . 4}$ \\
\hline Profesional, técnico o afín & 3.3 & 1.9 & 2.7 \\
\hline No profesional, no técnico & 27.4 & 30.4 & 28.7 \\
\hline T.F.N.R. 2/ & 2.4 & 6.1 & 4.0 \\
\hline RESTO 3/ & 1.0 & 11.8 & 5.5 \\
\hline TOTAL & $\mathbf{1 0 0 . 0}$ & $\mathbf{1 0 0 . 0}$ & $\mathbf{1 0 0 . 0}$ \\
\hline
\end{tabular}

$1 /$ Incluye empleadores.

2/ trabajador familiar no remunerado.

3/ incluye trabajadores del hogar, practicantes, y otro.

Por otra parte, de acuerdo a una nueva metodología aplicada por el Instituto Nacional de Estadística e Informática ${ }^{12}$, señala que en el período 2004-2006, la incidencia de la pobreza disminuyó en 4,1 puntos porcentuales al pasar de 48,6\%, en el 2004, a 44,5\%, en el 2006. Esta disminución en los niveles de pobreza se observa en mayor proporción en Lima Metropolitana, en donde la pobreza disminuye en 6,7 puntos

11 MINISTERIO DE TRABAJO Y PROMOCIÓN DEL EMPLEO. Indicadores Laborales. Febrero 2007. Cifras preliminares a octubre de 2006 de la Encuesta de Hogares Especializados en Niveles de Empleo.

12 Instituto Nacional de Estadística e Informática- INEI: Informe Técnico Medición de la Pobreza 2004, 2005 y 2006. Julio de 2007. 


\section{César A. Sanabria Montañez}

porcentuales, pasando de 30,9\%, en el 2004, a 24,2\%, en el 2006. Esta reducción en los niveles de pobreza es consecuencia de un mejoramiento del ingreso y gasto de los hogares señala el INEI.

Asimismo, el INEI señala que en el año 2007 los niveles de pobreza siguieron decreciendo; así, "en el año 2007, el 39,3\% de la población del país se encontró en situación de pobreza, es decir, tenían un nivel de gasto insuficiente para adquirir una canasta básica de consumo, compuesto por alimentos y no alimentos. Esta proporción de pobres que tiene el país está conformado, por 13,7\% de pobres extremos, es decir, personas que tienen un gasto per cápita inferior al costo de la canasta básica de alimentos y $25,6 \%$ de pobres no extremos, es decir, personas que tienen un gasto per cápita superior al costo de la canasta de alimentos, pero inferior al valor de la canasta básica de consumo” ${ }^{13}$ (ver Gráfico N. ${ }^{\circ} 1$ ).

Gráfico N. ${ }^{\circ}$ 1. Perú: Incidencia de la pobreza total en porcentaje, años 2004-2007

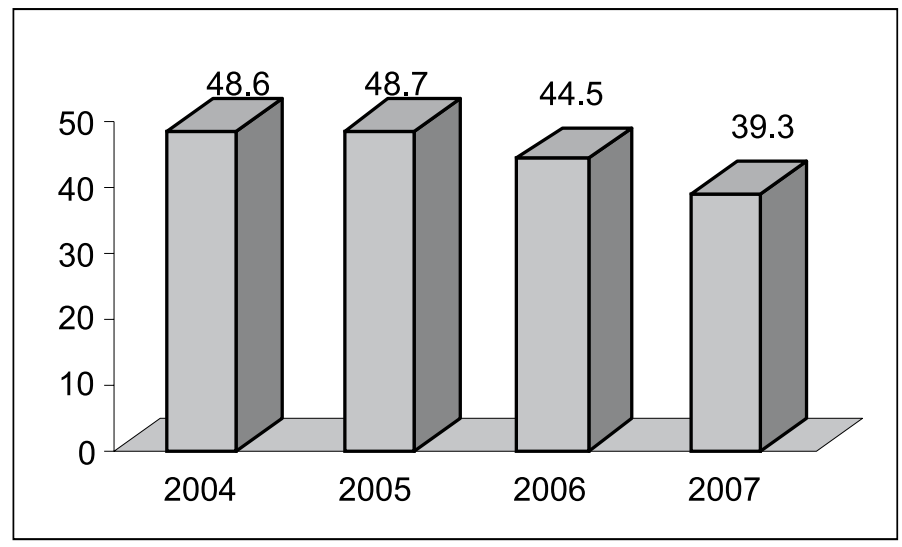

Fuente: INEI. Informe Técnico 2007, Informe 2006.

Las cifras presentadas por el INEI señalan que entre el 2006 y 2007 existe una disminución de la tasa de pobreza en 5,2 puntos porcentuales, al haber pasado de una incidencia de $44,5 \%$ al $39,3 \%$. Un tema importante es la extrema pobreza que se redujo en 2,4 puntos porcentuales, pasando de $16,1 \%$ a $13,7 \%$.

13 Instituto Nacional de Estadística e Informática- INEI: Informe Técnico: La pobreza en el Perú en el año 2007. 


\section{Marco para el análisis de la seguridad social en el Perú}

Señala, asimismo, el INEI: "Al analizar la incidencia de la pobreza por áreas de residencia y regiones naturales, se constata que el promedio nacional oculta situaciones de contraste. Mientras que la incidencia de la pobreza era de 25,7\% en las áreas urbanas, en las áreas rurales del país es de 64,6\%"14. Que señala la continuada exclusión del área rural a los beneficios del crecimiento económico.

Luego, se confirma que por región natural, la pobreza alcanzó, en el año 2007, al 22,6\% de la población de la Costa; siendo 37,5 puntos porcentuales menos que la pobreza de la Sierra $(60,1 \%)$ y 25,8 puntos porcentuales menos que en la Selva $(48,4 \%)$. Por dominios geográficos, en la Sierra rural, el 73,3\% de su población se encuentra en pobreza; seguida por la Selva rural, con 55,3\%; Selva urbana, con 40,3\%; Costa rural, con 38,1\%; Sierra urbana, con 36,3\%; Costa urbana, con 25,1\%; y Lima Metropolitana, con 18,5\% de pobres.

La incidencia de la extrema pobreza, por área de residencia, continúa concentrándose en el área rural. Así, mientras que en Lima Metropolitana el 0,5\% de su población son pobres extremos; en el área rural, dicha situación afecta al 32,9\% de su población.

Todo ello evidencia que la traslación del crecimiento logrado en la economía hacia los sectores sociales es lento, debido a las causas profundas de la pobreza en el Perú.

\section{EL CONTEXTO DEMOGRÁFICO ${ }^{15}$}

Un aspecto importante en el desarrollo económico social es el contexto demográfico y la dinámica poblacional, pues "los procesos demográficos determinan el tamaño y la estructura por edades y sexo de la población total y su evolución, condicionando, así, la composición de la fuerza laboral y los factores asociados a la productividad y niveles de ingresos" "16; asimismo, determinan las estrategias a seguir en términos de desarrollo social y las necesidades de recursos para hacer frente a las necesidades provisionales.

Así, en la sociedad peruana, la tasa de crecimiento poblacional viene disminuyendo notoriamente desde 1980 , de $2,4 \%$ a $1,7 \%$ anual en el año 2003. Pese a ese consi-

14 Instituto Nacional de Estadística e Informática- INEI: Informe Técnico: La pobreza en el Perú en el año 2007.

15 Este ítem está escrito a partir del estudio de ARAMBURÚ, Carlos y MENDOZA, María Isabel. "La población peruana: retos y perspectivas". En: Revista CIES Análisis de Políticas. Lima, 15/ 12/ 03.

16 ARAMBURÚ, Carlos y MENDOZA, María Isabel. "La población peruana: retos y perspectivas". En: Revista CIES Análisis de Políticas. Lima, 15/ 12/ 03 


\section{César A. Sanabria Montañez}

derable descenso, la población total ha seguido creciendo, de 17,3 millones a principios de 1980 a 27,3 millones en 2006. Se estima que en el año 2020, la población peruana sea de 31,3 millones de personas ${ }^{17}$.

"La población peruana está en plena transición demográfica, debido a la disminución de la mortalidad y la fecundidad, lo que influirá en la estructura de edades del país en los próximos años. En el futuro cercano, la población que crecerá más entre 2000-2005 será la adulta de 40-59 años (782 mil personas), seguida de la de 30-39 años (514 mil). Asimismo, en los próximos 15 años, habrá un envejecimiento de la población" ${ }^{18}$.

Por ello, del diagnóstico de la forma cómo viene evolucionando la estructura de edades en el Perú "... se aprecia claramente que uno de los grandes retos que enfrentará el país, en el mediano y largo plazo, será asegurar un sistema de seguridad social capaz de mantener a una población en proceso de envejecimiento" ${ }^{19}$. Y si tenemos en cuenta que existe un descenso en la fecundidad que llevará a un decrecimiento demográfico, ello implicará una mayor dependencia de los adultos mayores en relación con la población económicamente activa, que en términos de aportación a la seguridad social, recaerá en los trabajadores formales (aportantes al seguro de salud y de pensiones) y se verá agravada por los altos índices de informalidad existentes en el campo laboral. Asimismo, la existencia de una menor proporción de jóvenes podría repercutir desfavorablemente en los niveles de ahorro del país, y reducir las posibilidades de transferencias de recursos al interior de las familias ${ }^{20}$.

Asimismo, existen tres factores demográficos principales, que han dado forma a la actual distribución de edades y principales tendencias laborales del país. a) la ampliación de la brecha entre las tasas de natalidad y mortalidad que se dio entre las décadas de 1960 y 1980, b) la incorporación de la mujer en la fuerza laboral, y c) la distribución de la población y la consecuente reorganización de recursos y actividades.

Resulta indudable que en los años venideros, las tendencias demográficas, presentarán una serie de grandes desafíos para las políticas públicas a ejecutarse. Si bien la

17 INSTITUTO NACIONAL DE ESTADÍSTICA E INFORMÁTICA-INEI. Nuevas Proyecciones Nacionales de Población del Perú por Departamentos, Urbano y Rural y Sexo 2005 a 2020. Diciembre 2006.

18 ARAMBURÚ, Carlos y MENDOZA, María Isabel. Op. cit.

19 Ibídem.

20 Para mayor detalle de edades y fecundidad, ver en Aramburú y Mendoza. Op. cit. 


\section{Marco para el análisis de la seguridad social en el Perú}

tasa de crecimiento poblacional ha venido descendiendo en las últimas décadas, y ello comúnmente se asocia a mayor bienestar económico y social para un país; actualmente, se traducen en una fuerza laboral en explosión con bajos niveles de productividad, un paulatino envejecimiento de la población (Ver Gráfico N. ${ }^{\circ} 2$ ) y una acentuación de las brechas entre los niveles de desarrollo entre regiones.

\section{Gráfico N. ${ }^{\circ} 2^{21}$}
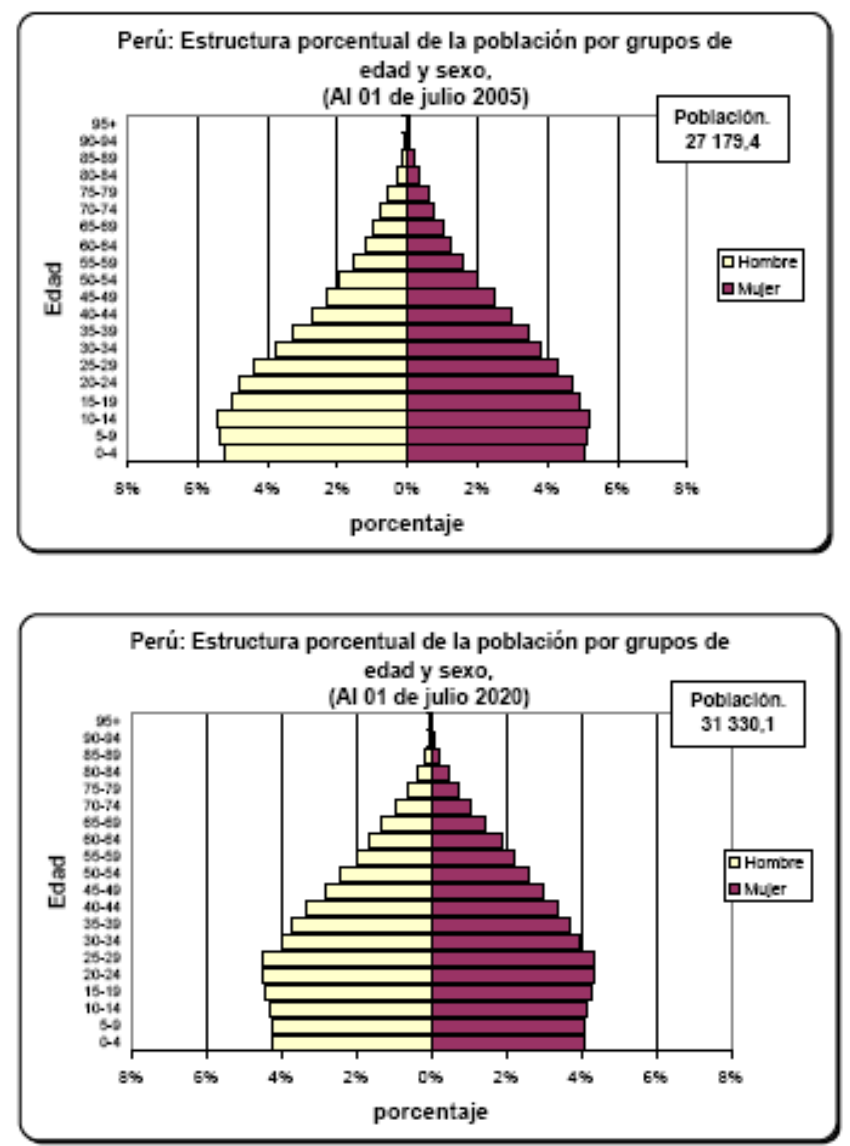

21 Fuente: INEI. Nuevas Proyecciones Nacionales de Población del Perú por Departamentos, Urbano y Rural y Sexo 2005 a 2020. Diciembre de 2006. 


\section{César A. Sanabria Montañez}

Finalmente, la heterogeneidad de los diferentes ámbitos geográficos y las distintas realidades de las localidades peruanas, posiblemente requerirán reformulaciones y mayor focalización de la política social, de manera que se atiendan las necesidades específicas a cada realidad regional. Asimismo, cabe señalar que las políticas de Estado, en términos de seguridad social y previsión social, implicarán un mayor esfuerzo en las zonas más pobres y excluidas de la población.

\section{LA SEGURIDAD SOCIAL EN PERÚ}

Desde la perspectiva de la seguridad social contributiva, la Seguridad Social en el Perú nace en agosto de 1936, cuando se da la Ley N. ${ }^{\circ} 843$, que crea el Seguro Social Obrero Obligatorio, cuya finalidad era de cubrir las pensiones y proteger la salud y maternidad de los obreros con carácter obligatorio; que, en términos prácticos, se inició la cobertura solo de salud de los obreros y no incluía a las esposas. Luego se incorpora, en 1948, mediante la Ley N. ${ }^{\circ} 10902$, a los empleados, se incluye entonces a los empleados y servidores públicos, y se amplía la cobertura por maternidad a la esposa del trabajador.

En 1961 y 1992, se amplían las coberturas a los obreros, empleados y servidores públicos, en el sentido de formar fondos de jubilación que cubra, vejez, viudez y orfandad.

En el año 1973, se crea el Sistema Nacional de Pensiones, busca unificar el sistema de pensiones, planteándose una jubilación a los 60 años para hombres y 55 años para mujeres, se otorga las prestaciones de pensión: de invalidez, de jubilación, de viudez, orfandad y ascendientes, y un capital de defunción. Ese mismo año, 1973, se crea el Seguro Social del Perú que fusiona el Seguro Obrero y el Seguro Social del Empleado, formándose una única gestora de la seguridad social peruana.

La organización del sistema de seguridad contributivo en el Perú fue como señalamos, fragmentado $y$, posteriormente se ha ido unificando, y en el tiempo se buscó darle autonomía financiera y -sobre todo- económica, así, “...mediante la creación del Régimen de Prestaciones de Salud, en 1979, se amplia la cobertura de las prestaciones al cónyuge y los hijos hasta los 18 años de edad. Finalmente, se crea en 1980 el Instituto Peruano de Seguridad Social (IPSS), con el fin de administrar los sistemas de pensiones y de salud y con la intención 


\section{Marco para el análisis de la seguridad social en el Perú}

de darle autonomía económica, lo que no se logra. Cubre tanto a obreros como a empleados" 22 .

Luego de la década de los 80 y de la hiperinflación en la economía peruana, el Estado estuvo más debilitado, en términos económicos sus ingresos habían decrecido, ante la recesión con inflación, lo cual debilitó más aún la sostenibilidad financiera de la seguridad social contributiva existente a la fecha; asimismo se dio inicio a un proceso de liberalización de la economía, en el que se incluyeron cambios estructurales, se plantearon una serie de reformas en todos los sectores, en el que no estaba ausente el sistema de seguridad social contributivo. Así, primero se dan unos pasos en la seguridad social en salud que finalmente no cuaja (se dio el Decreto Ley $\mathrm{N}^{\circ} 718$ que creo el Sistema Privado de Salud en el año 1991), y se creó el Sistema Privado de Pensiones en el año 1991 (D. Ley No 724) que empieza a funcionar en el año 1993.

La seguridad social en salud, recién en el año 1997, a través de la Ley de Modernización de la Seguridad Social en Salud, establece la participación del sector privado en el campo de la seguridad social en salud, de modo que señala que su participación es complementaria a través de las Empresas Prestadoras de Salud y se crea la

Superintendencia de Entidades Prestadoras de Salud -SEPS- como órgano regulador y supervisor de las EPS; y en el año 1999 se da la Ley de Creación del Seguro Social de Salud-EsSalud, que cambia el nombre al Instituto Peruano de Seguridad Social.

\section{EL SISTEMA DE SEGURIDAD SOCIAL EN SALUD}

La ley de modernización de la seguridad social en salud, fue un inicio de la participación privada en este campo, esta Ley señalaba que las personas que mediante votación laboral optarían por trasladarse a una EPS, tendrían derecho a una atención en la capa simple, quedando a cargo de la atención de la capa compleja el Seguro Social de Salud (EsSalud y ex Instituto Peruano de Seguridad Social).

Los aportes que harían las personas que opten por este régimen de atención para la capa simple, serían de dos maneras, mediante el traslado del $25 \%$ del total de su

22 VERDERA, Francisco. Seguridad social y pobreza en el perú: una aproximación. Documento de trabajo N. ${ }^{\circ}$ 84. IEP Instituto de Estudios Peruanos - Serie Economía. 1996. 


\section{César A. Sanabria Montañez}

aporte (la contribución a salud es del $9 \%$ del total de su remuneración o salario) y a través de mecanismos de copago.

A la fecha, existen tres Empresas Prestadoras de Salud, que tienen vinculación con 608 empresas que desarrollan actividades de atención. Las EPS empezaron a funcionar en el año 1999, han tenido un crecimiento limitado de cobertura de afiliaciones, cercana a 500000 personas. Si bien es cierto que existe una demanda potencial de extender el mercado de seguros potestativos para los trabajadores independientes de mayores ingresos en Lima Metropolitana y otras grandes ciudades. En un cálculo que se hace, se estima en 440 mil nuevos afiliados ${ }^{23}$.

Asimismo, en términos del Seguro Social de Salud (EsSalud), se observa que la cobertura poblacional de esta organización se ha estancado, visto desde una perspectiva de evolución entre el año 1994 al año 2005; así, su cobertura, en el año 1994, alcanzó a $21 \%$ de la población; mientras que en el año 2000, alcanzaba al 19.7\% de la población; y en el año 2005, alcanzó al $17 \%$ de la población ${ }^{24}$. Esta información señala "el agotamiento de un modelo de seguridad social basado en el empleo declarado en planilla, en un país en que el patrón de crecimiento económico no descansa en el empleo estable sino en el informal, con lo que sólo el $16 \%$ de la PEA está cubierta por EsSalud" 25 .

Además, se señala que EsSalud muestra una sostenibilidad financiera amenazada, por cuanto el crecimiento del gasto es mayor que el de los ingresos, que se refleja en los siguientes problemas ${ }^{26}$ :

1. Los trabajadores aportantes a EsSalud presentan una tasa decreciente en la PEA.

2. Elevada morosidad y evasión acumuladas de los empleadores (público y privado).

3. EsSalud recibe la mayor proporción de carga de enfermedades crónicas de alto costo, por el envejecimiento de la población y la selección adversa.

23 MADUEÑO, M.; ALARCÓN, J.; SANABRIA, C. "Analisis de la brecha entre oferta y demanda de servicios de salud para la programación de la inversión sectorial de mediano plazo". Lima: Partners for Health Reformplus. ABT 2003.

24 PETRERA, M. y SEINFELD, J. Repensando la salud en el Perú. Universidad del Pacífico, CIUP - Proyecto Amares. Julio de 2007.

25 Ibídem. p. 140.

26 Ibídem, pp. 143 y 144. 


\section{Marco para el análisis de la seguridad social en el Perú}

4. Creciente gasto en remuneraciones y limitantes institucionales para avanzar en la especialización de funciones y modificar mecanismos de pago.

En la parte de gestión de la prestación también surgen problemas de:

a. Contradicción entre planes de cobertura de servicios de salud, se plantea contenido universal y se tiene ingresos limitados.

b. Existencia de costos no explícitos (colas de espera, fallas en la calidad).

c. Barreras de acceso para contener la demanda.

d. Caída de la producción de servicios de salud.

e. Modelo eminentemente curativo y concentrado en grandes hospitales.

\section{EL SISTEMA PREVISIONAL (ONP / AFP)}

La seguridad social en pensiones en Perú opera actualmente bajo un esquema paralelo en el que coexisten básicamente dos sistemas. El Sistema Público de Pensiones y el Sistema Privado de Pensiones. Así, pertenecen al Sistema Público los regímenes: a) Decreto Ley N. ${ }^{\circ} 19990$ (denominado Sistema Nacional de Pensiones-SNP), y b) Decreto Ley N. 20530 (denominado Cédula Viva), actualmente cerrado a nivel constitucional; y el régimen bajo el Sistema Privado de Pensiones (SPP), administrado por entidades privadas denominadas Administradoras Privadas de Fondos de Pensiones (AFP). Además, existen regímenes pensionarios menores, como el del personal militar y policial de las Fuerzas Armadas y Fuerzas Policiales; y la Caja de Beneficios Sociales del Pescador.

Régimen del Decreto Ley No. 19990 ó SNP. Es un sistema que beneficia a los trabajadores sujetos al régimen de la actividad privada (Ley N. 4916 - Decreto Legislativo $\mathrm{N}^{\circ}$ 728), a los obreros (Ley N. ${ }^{\circ} 8433$ ) y a los funcionarios y servidores públicos bajo el régimen de la actividad pública (Ley N. ${ }^{\circ}$ 11377/ Decreto Legislativo N. ${ }^{\circ} 276$ ) no incorporados al Régimen del Decreto Ley N. ${ }^{\circ} 20530$.

Es un sistema de reparto, el cual tiene como característica principal el otorgamiento de prestaciones fijas -sobre contribuciones no definidas- en valor suficiente para que la aportación colectiva de los trabajadores financie las pensiones. En la actualidad, este sistema es administrado por la Oficina de Normalización Previsional (ONP). 


\section{César A. Sanabria Montañez}

Las prestaciones que otorga el SNP son cinco: (1) jubilación; (2) invalidez; (3) viudez; (4) orfandad; y (5) ascendencia.

Régimen del Decreto Ley No. 20530. Se origina en leyes muy antiguas que concedían pensiones vitalicias a cargo del Tesoro Público a un grupo muy reducido de funcionarios del Estado, como recompensa por los servicios prestados. Con los años, el ámbito de aplicación de este régimen se fue ampliando, incluyendo más beneficiarios y con mayores beneficios.

En general, este sistema se encuentra desfinanciado y representa una constante presión en las finanzas públicas porque requiere del creciente apoyo del Tesoro Público tanto al financiamiento de la planilla como al valor de la pensión: Así requieren de transferencias anuales por S/. 6500 millones: $14 \%$ del Presupuesto Público ${ }^{27}$. El Subsidio al capital requerido es de S/. 0.85 y S/. 0.50 por cada S/. 1 de pensión del DL N. 20530 y 19990, respectivamente ${ }^{28}$.

Según las proyecciones de la Oficina de Normalización Previsional, este desfinanciamiento continuará en los próximos años, básicamente por la maduración del propio sistema y las tendencias demográficas.

Así, a futuro, esta situación actuarial no mejorará, básicamente porque arrastra pensiones que -en su momento- no fueron ajustadas, pero en la medida que el manejo sea similar, el costo estará acotado. Según el último estudio de pasivo actuarial elaborado por la ONP, se establece que el importe de este, alcanza a US\$18 487 millones, al 2006.

Este pasivo se distribuye en los US $\$ 9390$ millones que representa el valor presente de las pensiones que el Estado asume con los actuales 448 mil pensionistas, hasta la muerte del último beneficiario; y los US\$ 9097 millones que representa el déficit que asumirá el Estado, debido a que los aportes no alcanzarán para cubrir las pensiones de los asegurados (en valor presente).

Respecto de las tendencias demográficas, en el año 2050, habrá tres veces más ancianos que en la actualidad (ver cuadro N. ${ }^{\circ} 2$ ).

27 Ministerio de Economía y Finanzas. Dirección General de Asuntos Económicos y Sociales. Los Sistemas de Pensiones en Perú. Junio de 2006.

28 Ibídem. 


\section{Marco para el análisis de la seguridad social en el Perú}

Cuadro N. ${ }^{\circ}$ 2: Perú: porcentaje de población por grupos especiales de edad, Hipótesis de Crecimiento Medio. Años 1990-2050.

\begin{tabular}{|l|c|c|c|c|c|}
\hline \multirow{2}{*}{ Año } & \multicolumn{5}{|c|}{ Grupos especiales de edad } \\
\cline { 2 - 6 } & $\mathbf{0 - 4}$ & $\mathbf{0 - 1 4}$ & $\mathbf{1 5 - 2 4}$ & $\mathbf{1 5 - 6 4}$ & $\mathbf{6 5} \mathbf{y}+$ \\
\hline 1990 & 13,63 & 38,21 & 20,34 & 57,86 & 3,93 \\
\hline 1995 & 12,99 & 36,37 & 20,28 & 59,36 & 4,27 \\
\hline 2000 & 11,89 & 34,54 & 19,84 & 60,75 & 4,71 \\
\hline 2005 & 10,73 & 32,24 & 19,32 & 62,57 & 5,19 \\
\hline 2010 & 9,89 & 29,72 & 19,14 & 64,58 & 5,70 \\
\hline 2015 & 9,18 & 27,48 & 18,51 & 66,14 & 6,38 \\
\hline 2020 & 8,56 & 25,70 & 17,30 & 67,01 & 7,29 \\
\hline 2025 & 7,96 & 24,09 & 16,23 & 67,48 & 8,43 \\
\hline 2030 & 7,71 & 22,87 & 15,39 & 67,36 & 9,77 \\
\hline 2035 & 7,43 & 21,95 & 14,65 & 66,92 & 11,13 \\
\hline 2040 & 7,13 & 21,29 & 13,93 & 66,10 & 12,61 \\
\hline 2045 & 6,87 & 20,63 & 13,50 & 65,16 & 14,21 \\
\hline 2050 & 6,67 & 20,00 & 13,31 & 64,31 & 15,69 \\
\hline
\end{tabular}

Fuente: INEI. Peru: Estimaciones y proyecciones de población. 1950-2050.

URBANA - RURAL. 1970-2025. Boletín de Análisis Demográfico N. 35. 2001.

El Sistema Privado de Pensiones (SPP) se creó en la década de los 90 por la incapacidad del Estado de honrar sus compromisos previsionales al no haber administrado de forma correcta los sistemas previsionales a su cargo. Este sistema se sustenta en un esquema de cuentas individuales en el que cada trabajador financia su propia pensión. Así, en este sistema:

- Los beneficios no son definidos, pues el monto de pensión se obtiene al momento de la jubilación en función al fondo capitalizado, la rentabilidad y los aportes individuales realizados.

- No existe subsidio intergeneracional ni redistribución de recursos, pues rige el principio de equivalencia, es decir, los beneficios equivalen estrictamente a los aportes individuales rentabilizados. 


\section{César A. Sanabria Montañez}

\section{Su cobertura ha sido importante}

El número de afiliados al SPP ha ido aumentando. A 2006, el número de afiliados fue más de 3,3 millones y alcanza alrededor de $29 \%$ de la PEA total; el número de aportantes también ha crecido, pero en menor medida; totalizando, a 2006, 1,3 millones ( $11 \%$ de la PEA total).

Asimismo, el valor de fondo ha ido creciendo; y, a la fecha, supera los US\$10 000 millones; de los cuales, la mayor parte están invertidos en el país. El Fondo de Pensiones representa $13 \%$ del PBI y $67 \%$ del ahorro interno.

\section{Este sistema tiene a la fecha una serie de limitaciones ${ }^{29}$ :}

1. Diferencias de pensión con el SNP para algunos grupos de trabajadores, generando presiones por la desafiliación.

2. No opera bajo un mercado competitivo (en precios) y está altamente concentrado en pocas administradoras (posiblemente debido al reducido mercado laboral formal).

3. Tiene un elevado costo administrativo que desincentiva la afiliación (por ejemplo de independientes).

4. No ha alcanzado una adecuada cobertura, especialmente entre los más pobres.

5. Existe preocupación acerca del nivel de sus pensiones en el futuro, y especialmente las de los más pobres. Solo el $50 \%$ de su población cotiza, la densidad promedio de aportación no supera el $70 \%$, la tasa de aportación ha estado rezagada en $8 \%$ por casi 10 años, las expectativas de vida vienen siendo mayores, lo que llevará a autofinanciar pensiones por más tiempo.

6. Inexistencia del principio de solidaridad, lo cual ha hecho que el Estado también tenga que apoyar a este sistema y -peor aún-que este sea constantemente criticado generándose un descontento en determinados grupos de la población.

\section{MARCO CONCEPTUAL: LA SEGURIDAD SOCIAL}

Según la Organización Internacional del Trabajo (OIT) "La seguridad social implica acceso a la asistencia médica y garantía de ingresos, en especial en caso de vejez,

29 Ministerio de Economía y Finanzas. Dirección General de Asuntos Económicos y Sociales. Los Sistemas de Pensiones en Perú. Junio de 2006. 


\section{Marco para el análisis de la seguridad social en el Perú}

desempleo, enfermedad, invalidez, accidentes en el trabajo o enfermedades profesionales, maternidad, o pérdida del principal generador de ingresos de una familia" ${ }^{30}$.

Y es que de por medio está el riesgo, de enfermarse, perder el empleo, de la cesantía, de la invalidez; pues las personas estamos siempre expuestas a estos riesgos y los costos pueden ser altos, que incluso pueden poner en peligro el patrimonio acumulado de la persona o su familia; entonces, desde la perspectiva de la teoría de seguros, sería aconsejable transferir estos riesgos a través de la compra de un seguro; sin embargo, quien podrían acudir a esta práctica sería solo la población de mayores ingresos, quedando desprotegidos los grupos de ingresos medios e ingresos bajos.

La razón está en que los de mayor ingreso velan por la protección de su patrimonio, mientras que el resto de la población, con escaso o nulo patrimonio, prefiere quedar a merced de las circunstancias y la caridad del resto, ello en caso que el siniestro se materialice. De modo que, conciente o inconscientemente, la persona transfiere su riesgo a los otros miembros de la sociedad, quienes actuarán motivados por la caridad o mediante sistemas que descansen en el financiamiento estatal, asumiendo así los costos toda la sociedad. Entonces, a la luz de la experiencia del desarrollo de las sociedades, la solución ha sido establecer mecanismos de seguro compulsivo, que ha permitido abordar socialmente los riesgos involucrados ${ }^{31}$, creándose así los sistemas de seguridad social.

Desde la perspectiva económica, la seguridad social, entonces, es producto de un fallo en el mercado, dada la incapacidad del mercado para asignar de modo eficiente los recursos, ello debido a la escaso acceso al empleo formal, a la distribución desigual del ingreso y a la asimetría de la información en el mercado de seguros privados, por lo que se justifica económicamente que el Estado intervenga este mercado, abordando socialmente los riesgos.

Y es en lo que se refiere a la salud de las personas, está clara la necesidad de su recuperación y su posterior inserción en el mercado laboral, pues siguiendo el esquema de que una persona sana puede estudiar, trabajar, producir, tener ingresos, consumir y,

30 http://www.ilo.org/global/Themes/Social_Security/lang--es/index.htm

31 MOWBRAY, A., BLNACHARD, R. y WILLIAMS, C. Insurance: Its Theory and Practice in the United States, 6a ed. Krieger Pub. Co., Huntington, N. Y. 1979. 


\section{César A. Sanabria Montañez}

por tanto, lograr el bienestar, el riesgo de perder la salud implica un riesgo alto y altos costos. La economía señala la existencia de enfermedades catastróficas; es decir, en aquellas personas que no tienen una protección por su salud, se le presenta una enfermedad de tipo crónico (degenerativo o que incapacita para el trabajo), esta se convierte en una catástrofe económica para la familia, pues deberá sacrificar incluso los activos y propiedades para hacerla frente.

Lo mismo ocurre en lo referido al desempleo y la cesantía o la finalización de la vida productiva de la persona, el riesgo de su ocurrencia está siempre presente, por lo que debe haber una posibilidad de disponer de ingresos para la vida misma; esto ha constituido desde siempre una preocupación del ser humano. No importa que tales ingresos hayan sido o sean en dinero o en especie, o bien parcialmente de una forma y parcialmente de otra. Las sociedades entonces ha organizado un sistema que garantice y cubra a una mayor población con un abordaje social, es decir, a través de un sistema de seguridad social.

"De la cobertura de riesgos librada a la voluntad individual se derivó, a consecuencia de la evolución histórica y social, el concepto de seguro gremial o colectivo, por el cual un grupo de personas, regularmente constituido por quienes ejercían una cierta clase de labores, proveía fondos para la atención de aquellos mismos riesgos antes descritos, aunque alterando el principio de capitalización individual por el de capitalización colectiva. Un conjunto de corrientes y movimientos sociales tales como el laborismo, el cooperativismo, el socialismo, las orientaciones de raíz cristiana o más generalmente religiosa, signadas por un borroso anhelo común de solidaridad, se expresaban sucesiva o concomitantemente en el plano de la cultura y de las ideas, y daban sustento a la racionalización de aquellos procesos" ${ }^{32}$.

Así, el seguro social contributivo se relaciona esencialmente con un sujeto: el trabajador. Es a este sujeto a quien se le reconocen ciertos derechos y se le imponen determinadas obligaciones. La idea matriz parte de algunos supuestos apropiados a las circunstancias de lugar y tiempo histórico en las cuales el seguro social es instituido"33. Así, en el mejor de los casos, en el pleno empleo, la seguridad social abarcará a toda la población, bajo el entendido que el trabajador formará parte de una familia y que en la mayoría de casos es el jefe, su familia estará cubierta por el seguro social.

32 MARTÍNEZ VILLALBA, Luis José. "Panorama de la Seguridad Social en América Latina". CIESS.

33 Ibídem. 


\section{Marco para el análisis de la seguridad social en el Perú}

\section{A modo de Balance}

1. La economía peruana enfrenta una dura contradicción. Se tiene, en la actualidad, 7 años de crecimiento económico continuo con altas tasas que en promedio llegan a ser más del $6 \%$ anual; pero este crecimiento económico no ha llevado a mejorar la incidencia de la pobreza, el 39.3\% de la población se encuentra en situación de pobreza.

2. La pobreza en el Perú afecta más al sector rural (al 64.6\% de la población rural) y a las provincias.

3. Las tendencias demográficas, presentarán una serie de grandes desafíos para las políticas públicas en ellas las de seguridad social, la heterogeneidad de los diferentes ámbitos geográficos y las distintas realidades de las localidades peruanas; en términos de seguridad social y previsión social, implicará un mayor esfuerzo en las zonas más pobres y excluidas de la población.

4. La seguridad social en el Perú está limitada a la seguridad social en salud y seguridad social previsional (de pensiones). No alcanza un sistema que incluya al desempleo.

5. En lo que respecta a la seguridad social en salud, la Ley de modernización de la seguridad social en salud ha permitido la participación del sector privado para la atención de los casos simples de atención (capa simple), su participación es limitada y su crecimiento de afiliación está estancado; lo mismo ocurre con el Seguro Social de Salud (EsSalud), que ha tenido disminución en su cobertura (el año 2005 alcanzó al $17 \%$ de la PEA).

6. El seguro de pensiones, tiene dos actores importantes, el Sistema Público a través de la Oficina de Normalización Previsional y el del Sistema Privado, a través de las Administradoras de Fondos de Pensiones. El primero de los nombrados, desfinanciado y con limitaciones en su gestión; el segundo también muy limitado en ampliación de coberturas.

7. El empleo y sus formas, son importantes para la existencia de los seguros sociales, y allí estaría la explicación de las bajas coberturas, pues la forma de empleo determina la inserción en la seguridad social, que en el Perú se ve agudizado, pues más de la mitad de la PEA está en condición de trabajador informal. 


\section{César A. Sanabria Montañez}

\section{BIBLIOGRAFÍA}

Asociación Internacional de la Seguridad Social (2004). Actas de la 28 ${ }^{a}$ Asamblea General. Beijing.

Holzmann Robert y Hinz Richard (2005). Soporte del Ingreso en la Vejez en el Siglo Veintiuno: Una perspectiva internacional de los sistemas de pensiones y de sus reformas. Banco Mundial.

Levcovitz Eduardo (2006). Nuevas estrategias para extender la protección en salud. Desafíos de la protección social para la organización de los sistemas de salud en América Latina y el Caribe. Asociación Internacional de la Seguridad Social. Conferencia Regional Americana. Belize City, Belice, 28-31 de mayo de 2006.

Levinsky Richard, McKinnon Roddy (2005). Hacia una confianza renovada. Primera edición. Ginebra: Asociación Internacional de la Seguridad Social.

Mesa-Lago Carmelo (2006). Integración de políticas de protección social para extender la cobertura: El rol de las instituciones de seguridad social. Asociación Internacional de la Seguridad Social. Conferencia Regional Americana. Belize City, Belice, 28-31 de mayo de 2006.

Organización Internacional del Trabajo (2003). Perú: Propuesta de Programa Nacional de Trabajo Decente, 2004-2006. Informe preliminar.

Superintendencia de Entidades de Prestadoras de Salud. Boletín Estadístico. Al Primer Trimestre 2007. 